\title{
Spectra of superradiant lasers
}

Protsenko, I. E.; Uskov, A. V.; André, E. C.; Mørk, J.; Wubs, M.

\section{Published in:}

Advanced Solid State Lasers 2020

Link to article, DOI:

10.1364/ASSL.2020.JTh2A.19

Publication date:

2020

Document Version

Peer reviewed version

Link back to DTU Orbit

Citation (APA):

Protsenko, I. E., Uskov, A. V., André, E. C., Mørk, J., \& Wubs, M. (2020). Spectra of superradiant lasers. In Advanced Solid State Lasers 2020 [JTh2A.19] Optical Society of America (OSA). Optics Infobase Conference Papers https://doi.org/10.1364/ASSL.2020.JTh2A.19

\section{General rights}

Copyright and moral rights for the publications made accessible in the public portal are retained by the authors and/or other copyright owners and it is a condition of accessing publications that users recognise and abide by the legal requirements associated with these rights.

- Users may download and print one copy of any publication from the public portal for the purpose of private study or research.

- You may not further distribute the material or use it for any profit-making activity or commercial gain

- You may freely distribute the URL identifying the publication in the public portal

If you believe that this document breaches copyright please contact us providing details, and we will remove access to the work immediately and investigate your claim. 
See discussions, stats, and author profiles for this publication at: https://www.researchgate.net/publication/344729524

\section{Spectra of superradiant lasers}

Conference Paper · October 2020

DOI: 10.1364/ASSL.2020.JTh2A.19

\section{CITATIONS}

5 authors, including:

Igor E. Protsenko

- Russian Academy of Sciences

130 PUBLICATIONS 1,765 CITATIONS

SEE PROFILE

Some of the authors of this publication are also working on these related projects:

Project

PhC waveguides and lasers View project

Quantum plasmonics View project

\section{esper Mork}

Technical University of Denmark

607 PUBLICATIONS 10,541 CITATIONS

SEE PROFILE 


\title{
Spectra of superradiant lasers
}

\author{
I. E. Protsenko ${ }^{1,2, *}$, A. V. Uskov ${ }^{1}$, E. C. André2 ${ }^{2}$ J. Mørk ${ }^{2}$, and M. Wubs ${ }^{2}$ \\ ${ }^{1}$ Lebedev Physical Institute of RAS, Leninsky prospect, 53, Moscow, 119991, Russia \\ ${ }^{2}$ Department of Photonics Engineering, Technical University of Denmark, DK-2800 Kgs. Lyngby, Denmark \\ *protsenk@gmail.com
}

\begin{abstract}
:
The emission spectrum of a superradiant laser is calculated analytically using quantum Langevin equations. New sideband peaks and fine structure in the spectrum are predicted for lasers with strong relaxation oscillations. (C) 2020 The Author(s)
\end{abstract}

\section{Introduction}

Nowadays there is great interest in superradiant (SR) lasers, which combine an active medium with a large gain and a bad cavity [1], so that collective spontaneous emission into the lasing mode is significant. SR lasers have been realized with cold alkaline earth atoms [2], Rubidium atoms [3], and with quantum dots [4] as the active medium. SR lasers are less sensitive to cavity-length fluctuations, which is important for atomic clocks [2]. Superradiance leads to interesting collective effects, such as excitation trapping [3] and superthermal photon statistics [4].

An analytical description of SR lasers is complicated by the fact that their quantum noise is not a perturbation, the equations are nonlinear, and the polarization of the active medium cannot be adiabatically eliminated. We address these issues using an analytical approach in order to understand SR lasers and their spectra. We consider an example of single mode homogeneously broaden SR laser with active medium of $N_{0} \gg 1$ two-level emitters.

\section{Approach and basic equations}

We describe the laser by operators $\hat{a}$ of lasing mode, $\hat{v}$ of active medium polarization and $\hat{N}_{e}=N_{e}+\delta \hat{N}_{e}$ of population of upper lasing level with mean value $N_{e}$ and fluctuations $\delta \hat{N}_{e}$. We begin with well-known MaxwellBloch equations (MBE) for two-level laser, see for example, [5]. As a novel part of our approach, we rewrite MBE for quadrature components $\hat{\alpha}=2^{-1 / 2}\left(\hat{\alpha}_{x}+i \hat{\alpha}_{p}\right)$, for field and polarization operators $\hat{\alpha}=\{\hat{a}, \hat{v}\}$ and then introduce symmetric (S) $\hat{\alpha}_{+}$and anti-symmetric (A) $\hat{\alpha}_{-}$parts of quadratures $\hat{\alpha}_{x}=\hat{\alpha}_{+}+\hat{\alpha}_{-}$and $\hat{\alpha}_{p}=\hat{\alpha}_{-}-\hat{\alpha}_{+}$. We linearize the MBE above the lasing threshold and show that linear MBE can be used also near and below threshold, if population fluctuations are small. Finally we derive the basic equations of our approach

$$
\begin{aligned}
\dot{\hat{a}}_{ \pm} & =-\kappa \hat{a}_{ \pm} \pm \Omega_{0} \hat{v}_{ \pm}+\hat{F}_{a_{ \pm}} \\
\dot{\hat{v}}_{+} & =-\left(\gamma_{\perp} / 2\right) \hat{v}_{+}+\Omega_{0}\left(\hat{a}_{+} N+2 \sqrt{n} \delta \hat{N}_{e}\right)+\hat{F}_{v_{+}} \\
\dot{\hat{v}}_{-} & =-\left(\gamma_{\perp} / 2\right) \hat{v}_{-}-\Omega_{0} \hat{a}_{-} N+\hat{F}_{v_{-}} \\
\delta \dot{\hat{N}}_{e} & =-2 \sqrt{n}\left(\Omega_{0} \hat{v}_{+}+\kappa \hat{a}_{+}\right)-\gamma_{\|}(P+1) \delta \hat{N}_{e}+\hat{F}_{N_{e}} .
\end{aligned}
$$

Here $\kappa, \gamma_{\perp}$, and $\gamma_{\|}$are, respectively, decay rate of lasing mode, polarization and population of upper lasing level; $\gamma_{\|} P$ is pump rate; $\Omega_{0}$ is the vacuum Rabi frequency; $n$ and $N$ are mean photon number and population inversion, $\hat{F}_{\alpha}, \alpha=\left\{a_{ \pm}, v_{ \pm}, N_{e}\right\}$ are $\delta$-correlated Langevin forces $\left\langle\hat{F}_{\alpha}(t) \hat{F}_{\beta}\left(t^{\prime}\right)\right\rangle=2 D_{\alpha \beta} \delta\left(t-t^{\prime}\right), \alpha, \beta=\left\{a_{ \pm}, v_{ \pm}, N_{e}\right\}$, with diffusion coefficients $2 D_{a_{ \pm} a_{ \pm}}=\kappa / 2,2 D_{v_{ \pm} v_{ \pm}}=N_{0} \gamma_{\perp} / 4$ and $2 D_{N_{e} N_{e}}=\left(\gamma_{\|} / 2\right)\left[P\left(N_{0}-N\right)+N_{0}+N\right]$. We show that equations for A-parts $\hat{a}_{-}$and $\hat{v}_{-}$describe phase fluctuations, equations for S-parts $\hat{a}_{+}, \hat{v}_{+}$and for $\delta \hat{N}_{e}$ describe amplitude fluctuations of laser field and polarization.

The basic set of equations is linear and solved by Fourier-transform. We find Fourier-components $\hat{a}_{ \pm}(\omega)$, spectra $n_{ \pm}(\omega),\left\langle\hat{a}_{ \pm}(\omega) \hat{a}_{ \pm}\left(\omega^{\prime}\right)\right\rangle=n_{ \pm}(\omega) \delta\left(\omega+\omega^{\prime}\right)$ of ampliude and phase fluctuations, mean $n_{ \pm}=(2 \pi)^{-1} \int_{-\infty}^{\infty} n_{ \pm}(\omega) d \omega$ and population inversion $N$ determined from the energy conservation law $n=\left(\gamma_{\|} / 4 \kappa\right)\left[P\left(N_{0}-N\right)-N_{0}-N\right]$ with $n \equiv n_{-}(N)+n_{+}(N)-1 / 2$.

The linewidth of the phase fluctuation spectrum $n_{-}(\omega)$ leads to the well-known laser linewidth above threshold, $\gamma_{\text {las }}=(1 / 2)\left[2 \kappa \gamma_{\perp} /\left(2 \kappa+\gamma_{\perp}\right)\right]^{2} N_{\mathrm{sp}} h \omega_{0} / W_{\text {out }}, N_{s p}=\left(N_{0}+N\right) / 2 N_{t h}, N_{t h}=\kappa \gamma_{\perp} / 2 \Omega_{0}^{2} ; \omega_{0}$ is lasing frequency and $W_{\text {out }}$ is the output power. Neglecting population fluctuations, we recover another well-known result for laser linewidth below threshold differing by a factor of 2 from the above-threshold expression. The field power spectrum is

$$
n(\omega)=n_{-}(\omega)+n_{+}(\omega)-n_{+-}(\omega),
$$

where $n_{ \pm}(\omega)$ comes from commutation relations $\left\langle\left[\hat{a}_{+}(\omega), \hat{a}_{-}\left(\omega^{\prime}\right)\right]\right\rangle=n_{+-}(\omega) \delta\left(\omega+\omega^{\prime}\right)$. 


\section{Lasing spectra}

We take parameters for photonic crystal lasers with quantum dots: wavelength of lasing transition $\lambda_{0}=1.55 \mu \mathrm{m}$, background refractive index $n_{r}=3.3$, cavity mode volume $V_{c}=10\left(\lambda_{0} / n_{r}\right)^{3}$ with $N_{0}=100$ emitters; population relaxation rate $\gamma_{\|}=10^{9} 1 / \mathrm{s} ; \Omega_{0}=\left(d / n_{r}\right)\left[\omega_{0} /\left(h \varepsilon_{0} V_{c}\right)\right]^{1 / 2}$ with dipole momentum of lasing transition $d=10^{-28} \mathrm{Cm}$ so $\Omega_{0}=34 \gamma_{\|}$; cavity quality factor $Q=1.2 \cdot 10^{4}$ so $2 \kappa=100 \gamma_{\|}$. Figure show spectra of SR laser (a,c) and non-SR laser $(\mathrm{b}, \mathrm{d})$ for large $(\mathrm{a}, \mathrm{b})$ and for weak (c,d) pump. For large $P$ we found sideband peaks in the spectrum of SR
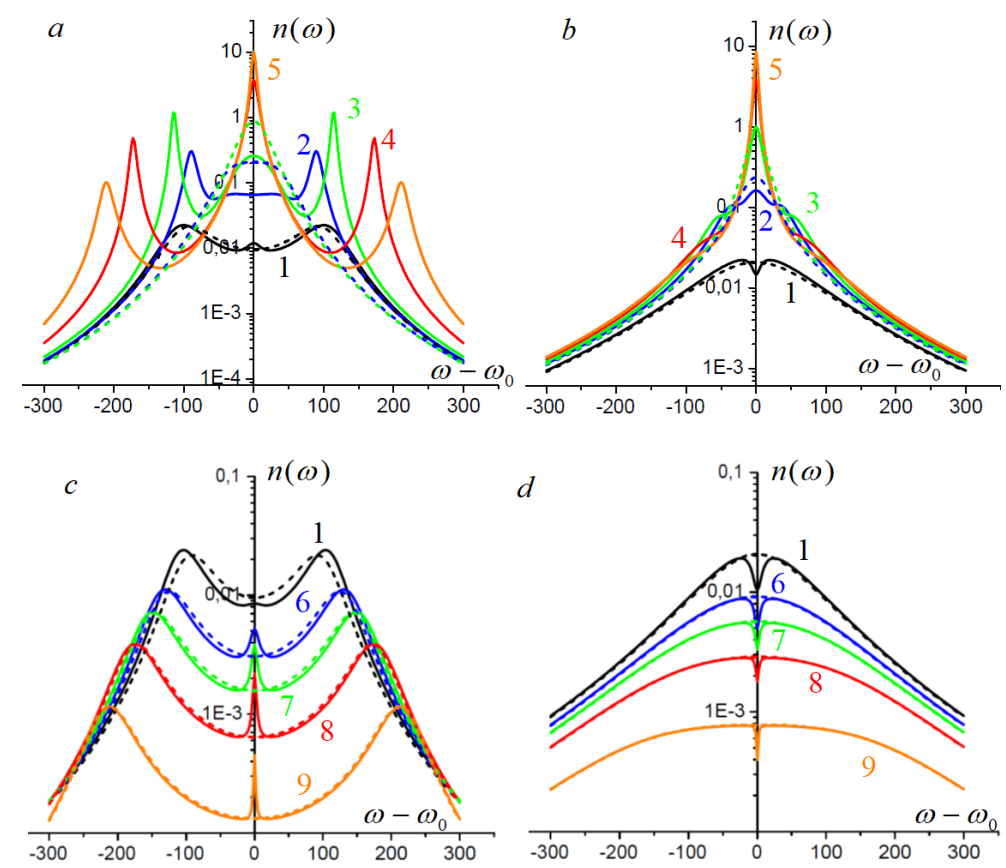

Fig. 1. Spectra of SR laser (a,c) with $\gamma_{\perp}=5 \cdot 10^{10} 1 / \mathrm{s}$ and $2 \kappa / \gamma_{\perp}=2$ and non-SR laser (b,d) with $\gamma_{\perp}=5 \cdot 10^{11} 1 / \mathrm{s}$ and $2 \kappa / \gamma_{\perp}=0.2$ for $P=2$ (curves 1); 8 (2); 16 (3); 28 (4), 40 (5), 1.12 (6), 0.8 (7), 0.48 (8) and 0.16 (9). Dashed curves, found without population fluctuations, have no sideband peaks at large pump and structures in the center at small pump.

laser caused by large population fluctuations and relaxation oscillations. Such sideband peaks are not resolved in spectra of non-SR laser, where population fluctuations are weak. For small $P$ we find a peak in the center of SR laser spectra exibiting collective Rabi spliting [5] and a dip for non-SR laser. This feature is due to interference in the linear and nonlinear (dependent on population fluctuations) parts of the polarization.

\section{Conclusion}

We present analytical quantum theory of spectra and fluctuations of superradiant (SR) and other lasers, above, near and below threshold retaining the polarization dynamics and collective effects. We reproduce well-known results for laser linewidth and predict new structures in the spectrum of SR lasers caused by population fluctuations.

\section{References}

1. C. Benkert, M. O. Scully, J. Bergou, L. Davidovich, M. Hillery, and M. Orszag, "Role of pumping statistics in laser dynamics: Quantum Langevin approach," Phys. Rev. A 41, 2756-2765 (1990).

2. J. K. Norcia, M. A. Thompson, "Cold-strontium laser in the superradiant crossover regime," Phys. Rev. X 6, 011025 (2016).

3. J. G. Bohnet, Z. Chen, J. M. Weiner, D. Meiser, M. J. Holland, and J. K. Thompson, "A steady-state superradiant laser with less than one intracavity photon," Nature 484, 78 (2012).

4. F. Jahnke, C. Gies, M. A $\beta$ mann, M. Bayer, H. A. M. Leymann, A. Foerster, J. Wiersig, C. Schneider, M. Kamp, and S. Höfling, "Giant photon bunching, superradiant pulse emission and excitation trapping in quantum-dot nanolasers," Nat. Commun. 7, 11540 (2016).

5. E. C. André, I. E. Protsenko, A. V. Uskov, J. Mørk, and M. Wubs, "On collective Rabi splitting in nanolasers and nano-LEDs," Opt. Lett. 44, 1415-1418 (2019). 